\title{
灵井动物群的埋藏学分析及中国北方旧石器时代中期 狩猎-屠宰遗址的首次记录
}

\author{
张双权 ${ }^{(1) *}$, 高星 ${ }^{(1)}$, 张乐 ${ }^{(1)}$, 李占扬 ${ }^{(2 *}$ \\ (1) 中国科学院古脊椎动物与古人类研究所人类演化实验室, 北京 100044 ; \\ (2) 河南省文物考古研究所, 郑州 450000 \\ * 联系人, E-mail: lizhanyang2621@sina.com; zhangshuangquan@ivpp.ac.cn
}

2011-05-06 收稿, 2011-08-01 接受

中国科学院战略性先导科技专项(XDA05130302)、中国科学院知识创新工程重要方向项目(KZCX2-EW-QN110)和国家自然科学基金 $(40872115,40602006)$ 资助

\begin{abstract}
摘要 河南灵井许昌人遗址 2005 2006 年发掘出土了上万件动物化石与古人类文化遗物. 埋 藏学分析表明了古人类在这一动物群聚集、改造过程中的主导性地位. 基于动物属种分布及 丰度、死亡年龄模式、骨骼表面改造痕迹与骨骼单元分布等动物群特点, 并结合生态环境等 方面的证据, 灵井许昌人遗址被解释为旧石器时代中期的一处狩猎-屠宰遗址而非古人类的 居址或中心营地. 这一遗址中数量较多的人工石制品的出现, 可能反映了古人类在生存策略 和行为活动方面的计划性和前瞻性. 灵井许昌人遗址是迄今为止中国北方旧石器时代中期 唯- - 处经过系统埋藏学分析确认的狩猎-屠宰类考古遗址.
\end{abstract}

关键词

灵井许昌人遗址

旧石器时代

埋藏学

动物考古学

狩猎-屠宰遗址
在旧石器时代的绝大多数时段内, 狩猎采集都 是古人类经济形态的主要方式. 狩猎经济一般在人 类食谱总量中的地位相对较低, 但对古人类的体质 演化、行为适应、组织形式等产生了多方面的重要影 响 ${ }^{[1]}$. 一般情况下, 在狩猎活动成功之后(尤其是在 猎捕大中型哺乳类动物的时候), 人类往往在猎捕现 场即对猎物的尸体进行初步的肢解和处理以便进行 搬运. 事实上, 即使是在最早期的考古遗址中, 也有 充足的证据表明, 古人类会对那些无法完整搬运的 大型动物的尸体进行处理, 然后再选择某些特定的 部分搬运回中心营地 ${ }^{[2]}$; 民族学和民族考古学的资料 同样证明了这一点 ${ }^{[3,4]}$. 考古学家一般认为, 当面临 搬运选择的时候, 古人类往往会选择搬运一些相对 高营养值的骨骼单元及其附属肌肉组织等, 同时也 将不可避免地在狩猎肢解场所丢弃一些低价值的营 养组分, 如头骨、下领、掌跖骨等 ${ }^{[3,4]}$. 这些因古代狩 猎者的选择性搬运而遗留下来的动物骨骼以及他们
用于肢解猎物的石制品便是判断遗址功能以及狩猎屠宰活动存在与否的关键性证据.

在旧石器时代考古遗址出土动物群的早期研究 史上, 学者们用以判断狩猎-屠宰遗址的主要依据是 动物骨骼与石制品之间的空间共生关系 ${ }^{[5]}$. 然而, 古 人类活动仅仅代表了自然界诸多骨骼聚集和改造过 程中的一个片段; 在人类之外, 还有其他埋藏学营力, 如水流、食肉类动物、大型猛禽甚至啮齿类动物等都 有可能造成动物骨骼的大量富集 ${ }^{[6,7]}$; 当它们与古人 类的文化遗存, 如石制品等共同赋存时, 就有可能影 响到对于遗址性质的考古学判断 ${ }^{[8]}$. 进人二十世纪八 九十年代之后, 世界范围内的实验研究及生态观察 更是证实了传统做法的严重局限 ${ }^{[1,8]}$. 因此, 对于考 古遗址出土的大量动物骨骼而言, 埋藏学手段的介 人和渗透将是判断遗址形成过程、梳理自然营力与人 工作用、推测古人类行为模式及遗址功能与性质的先 决条件与依托 ${ }^{[6]}$. 民族学研究与实验观察业已表明,

英文版见: Zhang S Q, Gao X, Zhang Y, et al. Taphonomic analysis of the Lingjing fauna and the first report of a Middle Paleolithic kill-butchery site in North China. Chinese Sci Bull, 2011, 56: 3213-3219, doi: 10.1007/s11434-011-4718-2 
狩猎-屠宰场所的人类活动一般是通过猎杀、屠宰以 及敲骨吸髓等过程完成的, 因此在其中的动物骨骼 之上一般也会遗留一些人类行为造成的物理性损伤 痕迹, 例如切割痕和砍砸痕等. 在旧石器时代的考古 遗址中, 古人类肢解动物的行为也是久而有之, 距今 约 250 万年前的 Gona 遗址中已有古人类肢解猎物的 证据 ${ }^{[9]}$. 此外, 在肢解活动发生之后, 一般会在狩猎 者的生活营地发现更多营养值较高的骨骼部位(如营 养物含量较高的股骨等); 相反, 那些营养值相对较 低的骨骼部位(如营养物含量较低的掌跖骨等)则会 在狩猎或肢解场所更多的出现 ${ }^{[3,4]}$. 这些都将成为判 断考古遗址性质与功能的重要依据.

\section{1 遗址概况}

灵井许昌人遗址位于河南省许昌市西北约 $15 \mathrm{~km}$ 的灵井镇西侧, 地理坐标为 $34^{\circ} 04^{\prime} \mathrm{N}, 113^{\circ} 41^{\prime} \mathrm{E}$, 海拔 高度 117 m. 2005 2006年, 河南省文物考古研究所对 该遗址进行了新的发掘与研究, 在近 $300 \mathrm{~m}^{2}$ 的范围 内出土石制品近万件以及包括部分骨器在内的动物 骨骼化石万余件 ${ }^{[10]}$, 包括啮齿类 2 种, 食肉类 3 种, 长鼻类 1 种, 奇蹄类 4 种, 偶蹄类 8 种, 共计 18 个属 种 ${ }^{[11]}$. 李占扬等人 ${ }^{[11]}$ 根据生物地层判断这一动物群 的时代应与许家窑动物群的时代相似, 为晚更新世早 期, 其年龄约在 $100 \mathrm{ka}$ 左右. 周力平等人完成的初步 光释光测年结果表明, 埋藏古人类头骨化石层位的时 代应该在 80 100 ka 之内, 甚至可能稍大于 10 万年 (个人交流). 这一时间段与欧洲旧石器时代中期(UP) 或非洲石器时代中期(MSA)大致相当 (在中国旧石器 文化演化序列中, 也有学者将其视为旧石器时代早 期向晚期的过渡阶段 ${ }^{[12,13]}$ ). 灵井动物群的埋藏学分 析表明, 原始牛 (Bos primigenius) 和普通马 (Equus caballus) 是该动物群中居于绝对数量优势的 2 个属 种 ${ }^{[14]}$.

\section{2 灵井动物群的埋藏学特点}

古人类、食肉类动物、啮齿类动物、大型猛禽、 流水作用, 包括自然沉积等都可以导致考古遗址中 大量动物骨骼的聚集 ${ }^{[6,7]}$. 在灵井动物群的骨骼聚集 营力方面, 首先可以排除的是啮齿类动物. 这一判断 主要来源于动物群中低至 $0.06 \%$ 的具有啮齿类啃咬 痕迹的动物骨骼比例 ${ }^{[14]}$. 通常在豪猪等啮齿类动物 聚集而成的动物群中，大约 $20 \%$ 80\%的骨骼标本上
都会出现啮齿类动物的啃咬痕迹 ${ }^{[15,16]}$, 这与灵井动 物群中的相应数值差别显著, 无疑应是否定啮齿类 作用的有力证据. 此外, 对于旷野类遗址出土的动物 群而言，啮齿类动物一般也很难成为其主导的聚集 因素. 豪猪等啮齿类动物倾向于将大中型动物的骨 骼搬运到它们的巢穴中 ${ }^{[15]}$, 但是, 在现代生态学的 观察中, 目前还没有发现它们在旷野环境中大量聚 集骨骼的任何记录 ${ }^{[16]}$; 此外，一般情况下，啮齿类动 物搬运的动物骨骼其风化程度大都比较强烈 ${ }^{[6]}$, 这些 都与灵井动物群形成了鲜明对照.

猫头鹰等猛禽也有在其巢穴中聚集大批动物骨 骼的习性. 但是, 与其生态地位相适应的是, 猫头鹰 等猛禽一般是以啮齿类、兔形类、其他鸟类以及小型 灵长类作为其主要猎物来源的 ${ }^{[6,17]}$, 这一动物属种组 成与灵井动物群的当前面貌差别明显; 此外, 在骨骼 表面的胃酸腐蚀特征方面, 灵井动物群中此类标本 的缺失也截然有别于典型的猛禽类聚集的动物骨骼 堆积 ${ }^{[18,19]}$. 因此, 在灵井动物群的聚集营力方面, 猫 头鹰等大型猛禽类的可能性无疑也是可以排除的.

流水作用可以导致动物骨骼的大量聚集 ${ }^{[6,7]}$. 然 而, 对于考古遗址出土的动物群而言, 流水作用的最 大体现通常并非在于动物骨骼材料的聚集方面，而 是改造已经形成的动物群组合 ${ }^{[20,21]}$. 当然, 在一定的 水动力条件和适合的环境下, 流水携带的某些动物 骨骼也可以发生集中沉降, 从而形成动物骨骼的大量 聚集，例如山前地带的冲积扇等. 灵井遗址 $15.75 \%$ 的 动物骨骼标本上具有水流磨蚀的痕迹; 同时, 在原始 牛的骨骼单元组合方面, 肩胛骨的\%MAU 数值相对 较高(36.47). 上述证据表明, 灵井动物群或许存在一 定程度的水流改造的可能, 但却不会是一个因流水 作用而聚集的动物骨骼组合 ${ }^{[14]}$.

至于自然沉积, 往往是许多学者容易忽略的一 个环节. 事实上, Potts ${ }^{[22]}$ 及 Domínguez-Rodrigo 等人 ${ }^{[8]}$ 都已令人信服地证实了同类动物群的广泛存在. 然 而, 对于灵井动物群而言, 尽管其生态环境指示了自 然沉积的可能(湖滨、水塘附近等), 但是这一动物群 的动物属种组合及其年龄分布决定了它绝对不应该 是一个自然堆积的结果: 灵井动物群以大中型哺乳 类动物为优势种的特点与自然沉积下产生的以中小 型动物属种为主的组合面貌并不吻合; 此外, 灵井动 物群中的 2 种主要动物类型原始牛和普通马都是以 壮年个体占据数量优势的一种年龄组合，这也不同 
于自然情况下产生的以幼年和老年个体为主的年龄 组合特点 ${ }^{[14]}$. 在湖滨、水塘附近, 大型动物陷落于池 沼之中而无法自救也是可能造成大型动物骨骼聚集 的自然性因素之一 ${ }^{[23]}$. 但是, Haynes ${ }^{[24]}$ 对非洲象的多 年观察结果表明, 这一现象大多发生在老幼病残个 体的身上, 很少能够见到壮年或成年的非洲象会发 生这样的事情; Berger ${ }^{[25]}$ 对现代野马、Sinclair ${ }^{[26]}$ 对现 代水牛自然居群的观察也都证明了这一点. 因此, 灵 井动物群中两种大中型食草类动物的年龄特点 (成年 个体数分别占 $82.4 \%$ 和 $61.1 \%$ )否认了自然沉积的可 能性.

在食肉类作用方面(这是目前埋藏学研究最深 人、最详细的一个领域), 目前已知蕆狗、豹等动物 都可以在其栖息地或巢穴内聚集较为大量的动物骨

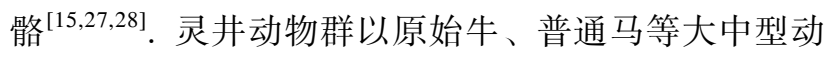
物种类占据数量优势 (最小个体数分别为 18 和 17), 同时仅有 $5.4 \%$ 的动物骨骼表面具有食肉类齿痕，表 明豹不会是灵井动物群骨骼聚集的主导因素, 至少 对于原始牛、普通马等大型动物而言, 豹的作用几乎 是可以忽略不计的. 䈓狗, 从目前生态研究以及考古 研究的结果来看, 在它们的巢穴中有时确实会有大

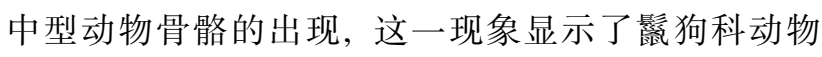
聚集大型动物 (尤其是食草类动物)骨骼的可能性. 然 而, 灵井动物群中低至 $5.4 \%$ 的食肉类齿痕标本比例, 以及瓺狗粪化石与骨骼表面胃酸腐蚀痕迹的缺失等

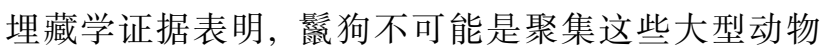
骨骼的主要因素 ${ }^{[14]}$ (图 1), 这里, 还需要说明一下,

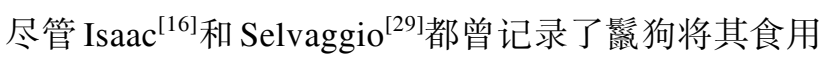
后的剩余动物骨骼 (包括其上附带的营养成分)暂时 “储存”在较浅的水池中而此后又经常忘记找回的这

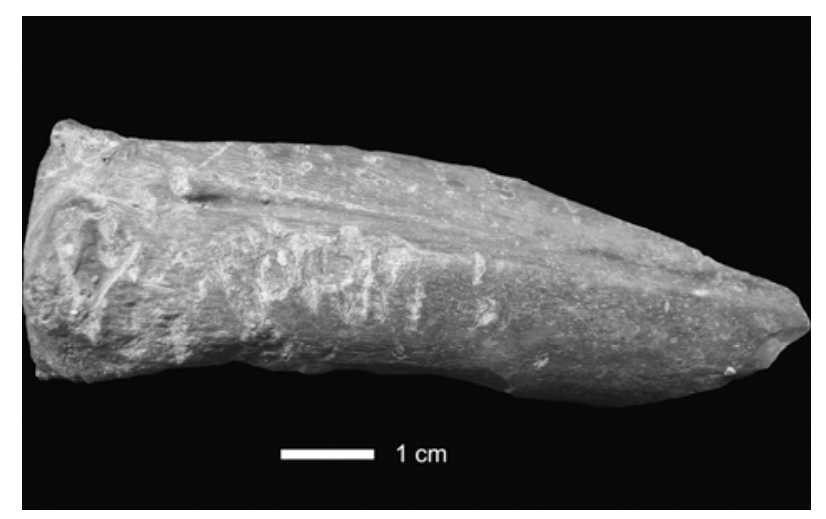

图 1 鹿掌骨上的食肉类齿痕
一习性(此时, 这些动物骨骼上往往少有食肉类齿痕 的产生; Selvaggio ${ }^{[29]}$ 甚至认为这也是造成早期动物 群聚集的原因之一), 但是, 此类记录依然无法解释 灵井动物群中以原始牛、普通马等大中型动物为主, 而且它们都是以壮年个体占据数量优势的这一现象.

总之, 在目前已知的能够大量聚集动物骨骼的 各种自然营力中, 无一可与灵井动物群的埋藏学记 录相吻合. 在自然营力之外, 古人类作用是导致考古 遗址中动物骨骼大量富集的另一主要因素 ${ }^{[6,7]}$. 灵井 动物群中的原始牛、普通马等 2 种动物都以壮年个体 占据数量优势(分别为 $82.4 \%$ 和 $61.1 \%$ ), 同时又有一 定比例(分别为 $17.6 \%$ 和 $33.3 \%$ )的幼年成员 ${ }^{[30]}$. 这种 以壮年成员为主要代表类群的动物死亡年龄模式在 考古遗址出土的动物群研究方面具有非常特殊的意 义, 它是古人类行为的独特体现. 动物考古学研究表 明, 旧石器时代中期的狩猎-采集人群往往选择猎取 动物居群中的壮年个体以追求营养效率的最大化, 这与后期考古记录中的以整个动物居群或其中的老 年或幼年个体为重点捕杀目标的人类行为特点有着 明显的差别 ${ }^{[14,30]}$. 灵井许昌人遗址出土的动物骨骼 中, 大约 $17.2 \%$ 的标本上都有切割痕、砍砸疤等古人 类行为痕迹的出现, 这一现象同样指示了古人类在 这一动物群形成过程中的主导性作用 ${ }^{[14]}$.

\section{3 遗址的功能与性质}

对于考古遗址出土的动物群而言, 遗址性质和 功能的不同将会导致动物群面貌以及古人类行为模 式方面的巨大差异 ${ }^{[3]}$. 由于旧石器时代旷野类遗址形 成过程的复杂性, 分辨遗址的性质与功能往往并非 想象的那么简单 ${ }^{[31,32]}$. 然而, 对于灵井许昌人遗址而 言, 却有足够的证据将其解释为旧石器时代中期古 人类的狩猎-屠宰遗址而不是他们的居址或“中心 营地”.

（i ) 动物属种的特点. 一般情况下, 当人类成 功猎取了较小体型的食草类动物时, 大多选择将其 完整地搬运回中心营地或者只是进行初步的野外肢 解 ${ }^{[4]}$. 因此, 在狩猎-屠宰遗址通常都有更多大中型 动物骨骼材料的遗留; 相反, 古人类的居址则以相对 较多的中小型动物为其特点 ${ }^{[33]}$. 这一点也在现代狩 猎采集群体中得到了证实 ${ }^{[4,34]}$. 灵井动物群以原始牛、 普通马为绝对优势种的事实与狩猎-屠宰遗址的特点 更为符合(图 2). 


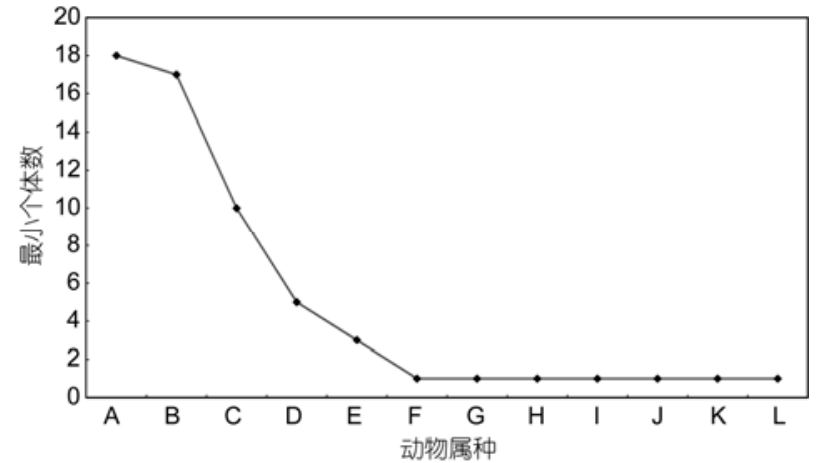

图 2 灵井动物群主要动物属种的最小个体数分布

A, Equus sp.; B, Bos primigenius; C, Megaloceros ordosianus(Cervus elaphus); D, Coelodonta antiquitatis; E, Procapra przewalskii; F, Dicerorhinus mercki; G, Pachycrocuta cf. sinensis; H, Palaeoloxodon sp.; I, Viverra cf. zibetha; J, Ursus sp.; K, Sus lydekkeri; L, Hydropotes pleistocenica

（ii）骨骼单元组合的证据. 当狩猎者面临搬运 选择的时候, 他们总是希望能够最大限度地搬运更 多的营养物质(包括附着有肌肉、骨髓和油脂等多种 营养成分的动物骨骼), 同时也将不可避免地在狩猎 场所丢弃一些低价值的营养物质及其所附着的动物 骨骼部位(单元). 因此, 在狩猎者的生活营地中通常 都会发现更多的高营养值的骨骼部位; 相反, 那些营 养值相对较低的骨骼单元则会在猎物的死亡地或者 狩猎场所更多的出现 ${ }^{[3,4]}$. 较之于脊椎、肋骨等, 动物
骨骼中的附肢骨，尤其是其中上部骨骼单元传统上 都被看作是更有营养价值的部分. 也就是说, 对于考 古类遗址而言, 由于古人类搬运行为的影响, 狩猎屠宰遗址一般都会以动物的头盖骨、下领骨 (当然, 由于后期埋藏学作用的影响, 更多情况下见到的则 是上下领的牙齿)以及下部肢骨(掌骨、跖骨等)为主; 而古人类的居址或 “中心营地”则会以中上部肢骨(股 骨、肱骨、胫骨、桡骨、尺骨)等高营养值的骨骼材 料为主 ${ }^{[3,4]}$. 灵井动物群以动物的上下领牙齿以及下 部肢骨等低营养值的骨骼单元占据数量优势 (其 $\% \mathrm{MAU}$ 值分别为 100 和 38.38)的事实同样支持我们 将这一遗址解释为狩猎-屠宰遗址(图 3).

（iii）骨骼表面人工改造痕迹证据. 灵井动物群 中具人工切割痕的长骨标本中(在古人类行为的解释 方面, 大中型食草类动物的长骨类骨骼单元往往具 有更为明确的指示意义 ${ }^{[8]}$, 因此我们的讨论仅限于此 类标本), 食草类动物上部与中部肢骨的骨骼数量较 多, 分别占 $34 \%$ 和 $41 \%$; 相反, 属于下部肢骨的仅占 $25 \%$. 与此类似，对于同一长骨单元(如股骨)的不同 部位而言, 绝大多数标本上的切割痕都位于长骨的 骨干部位(185 件, 占相应标本总数的 $98.45 \%$ ), 而位 于远、近两端骨骺的相应骨骼数分别只有 2 件和 1 件 (分别占标本总数的 $1.06 \%$ 和 $0.53 \%$ )(图4). 这一分布
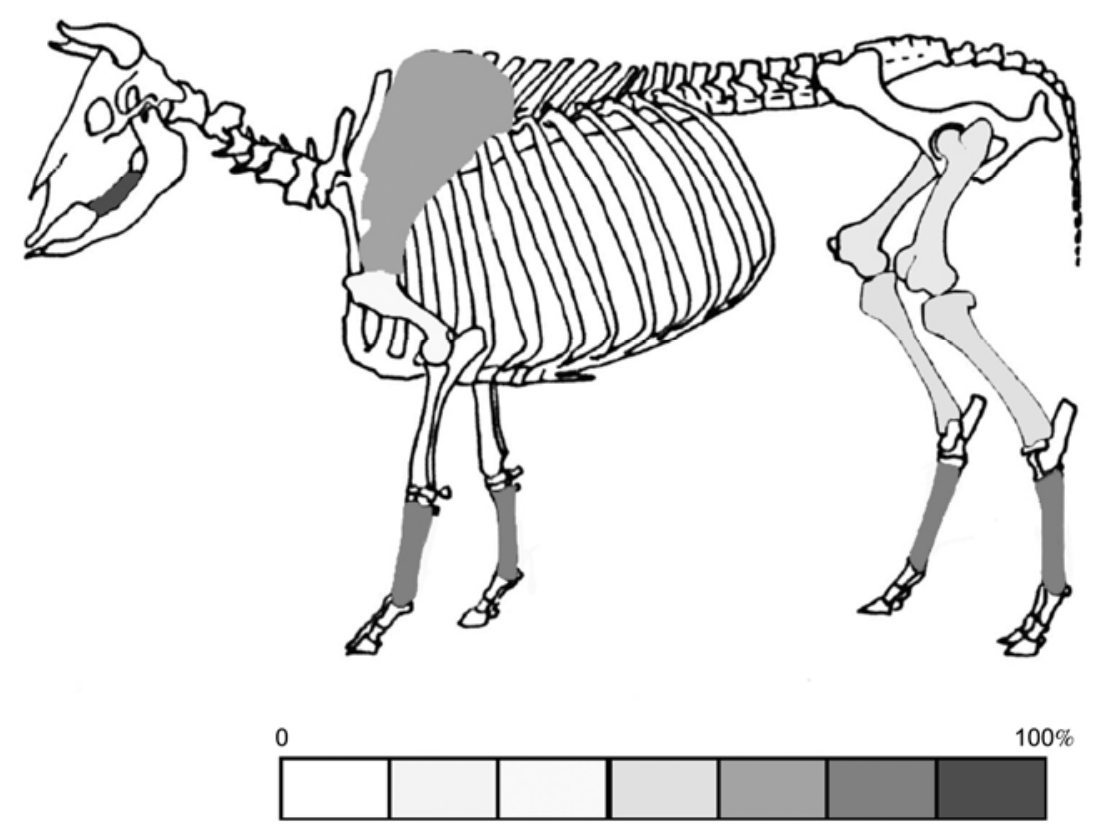

图 3 灵井动物群原始牛的骨骼单元分布(\%MAU) MAU, 最小骨骼单元数 ${ }^{[3,35]}$ 


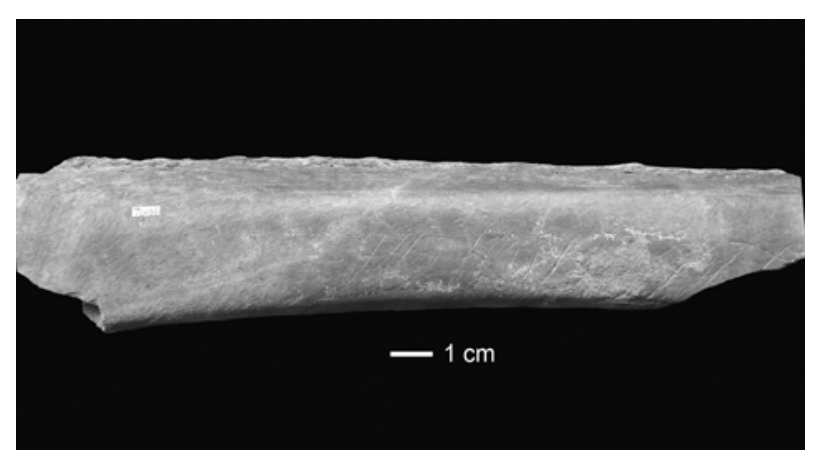

图 4 大型动物(等级 III 或IV)肋骨上的切割痕

规律与 Domínguez-Rodrigo ${ }^{[36]}$ 的实验结果及 Lupo 等 ${ }^{\left[{ }^{[37}\right.}$ 的民族学观察较为一致，表明灵井时期的古人 类是以割取动物长骨上的大量肌肉组织为其目标的; 他们不仅是遗址中大型动物的猎取者, 同时也是这 些肉食资源的初级利用者. 灵井动物群中人工切割 痕的出现及其分布规律强烈暗示了遗址中古人类狩 猎与肢解动物行为的存在. 更为重要的是, 灵井遗址 出土的骨骼标本上几乎没有刮削型切割痕(scraping mark)的出现, 这一粗放型的资源提取方式也与狩猎屠宰遗址中的古人类行为特点更为吻合.

（iv）遗迹、遗物及生态环境方面的证据. 在旧 石器时代的考古遗址中, 火塘是最具代表性的遗迹 现象之一; 尤其是在古人类较长时间活动的“居址” 类遗址中, 火塘往往较为常见. 相反, 对于狩猎-屠宰 遗址而言, 由于古人类的活动主要局限于猎取和肢 解各种动物, 因此一般缺乏“居址”中较为常见的火 塘以及与其密切伴生的烧骨等古人类遗物. 灵井遗 址至今尚末见到有关火塘的报道, 并且其中仅有的 数件 “烧骨”更有可能来源于沉积后的较为猛烈的自 然性过火事件, 而不是古人类以提取营养成分为目 标的相对轻微的 “灼烧”的结果 ${ }^{[14]}$, 这种现象与狩猎屠宰遗址的情况更为接近. 目前, 还没有对遗址出土 的近万件石制品进行使用与功能方面的研究, 但是, 动物骨骼标本的微痕观察结果已经初步揭示了古人 类对于遗址附近动物性资源的提取与加工行为 ${ }^{[38]}$.

在古生态环境方面, 灵井遗址中发现的龟类化 石以及原始牛化石的大量出现指示了水体环境的存 在; 同时，遗址堆积性质以及骨骼表面的生物化学腐 蚀痕迹也都指向了相对潮湿的环境, 表明灵井遗址 本身即处于湖滨环境. Binford ${ }^{[39]}$, Andresen 等人 ${ }^{[40]}$ 和 Pitts ${ }^{[41]}$ 等多位学者都已指出, 由于各种食肉类动物
的频繁出没，湖滨、河滩等生态环境并不适合古人类 的居住 ${ }^{[40,41]}$; 即使是在今天的狩猎采集群体中, “中 心营地”通常也不会被安置在距离水体很近的地方 ${ }^{[39]}$. Potts $^{[22]}$ 同样认为, 此类环境并不适宜古人类栖居, 因为他们丢弃在此的动物骨骼可能会招致大量食肉 类动物的夜间造访和侵扰. 总之, 灵井遗址的生态环 境几乎决定了它无法作为古人类居址的客观命运. 事实上，尽管许多被解释为古人类“居址”或“生活面” 的非洲早期人类遗址大都比较接近当时的河滨或湖 滨，但是其实际距离还是远大于灵井遗址的目前情 况, 比如, Koobi Fora 遗址距离湖边大约有 15 20 $\mathrm{km}^{[42]}$; 即使是在 FLK 和 FLK North 等遗址, 这一距 离也在 1 2 $\mathrm{km}$ 之外 ${ }^{[22]}$.

\section{4 讨论}

目前，动物考古学家对于旧石器时代狩猎-屠宰 遗址的认定，其前提条件即是埋藏学分析的介人以 及古人类狩猎、肢解或屠宰猎物的充分证据 ${ }^{[43]}$. 灵井 动物群的埋藏学特点表明，古人类作用是导致这一 动物群形成的主导性因素; 灵井遗址记录了远古人 类狩猎、屠宰大中型哺乳类动物的典型行为特征与生 存策略. 依照 Isaac 等人 ${ }^{[5]}$ 的经典模型, “B 类遗址”(即 狩猎-屠宰遗址) 的特点是某一种类的大型动物的单 个骨架以及数量较少 (一般为数件至数百件)的相应 石制品的组合. 与传统的狩猎-屠宰遗址相比, 灵井 动物群不仅在动物属种的多样性及动物个体数方面 远远超过了它的要求, 而且在伴生石制品方面, 这一 遗址也因其数量的极度丰富而与 “B 类遗址”严重不 符. 然而, 就在 Isaac 发表其论著后不久, 西方学者 就在考古记录中先后发现了这一传统定义的诸多不 足. Potts ${ }^{[22]}$ 认为, “B 类遗址”是将考古遗址出土的动 物群过于简单化处理的一种方案. 更多情况下，考古 遗址或者是在动物属种的多样性及动物个体数方 面 $^{[22]}$, 或者是在相关石制品的缺失(尽管遗址出土的 动物骨骼上可能还有人工切割痕的出现)方面 ${ }^{[41,44]}$ 往 往都与这一定义有着相当大的差别.

从遗址功能的角度分析, 灵井遗址无疑符合狩 猎-屠宰遗址的基本特征. 基于居群生态学等方面的 分析结果, 灵井动物群中多个动物个体的出现反映 了一种多期次的狩猎-屠宰活动相互“叠压”的过程 ${ }^{[14]}$. 这一现象与最近 Chazan 等人 ${ }^{[45]}$ 在以色列的发现较为 一致. Chazan 等人 ${ }^{[45]}$ 依照早期学者的观点, 将经典的 
“B 类遗址”称之为“单个动物尸体遗址”(single carcass sites), 而将具有多个动物骨架的遗址称之为“多个动 物尸体遗址”(multiple carcass sites). 在动物群及石制 品组合方面, Chazan 等人 ${ }^{[45]}$ 定义的“多个动物尸体遗 址”与 Issac 定义的“C 类遗址”(Type C sites, 即居址) 似乎更为接近一些. 然而, 相对于传统定义而言, “多 个动物尸体遗址”大大弱化了“C 类遗址”作为“居址” 的古人类行为方面的含义. 这里, 我们基本认可 Chazan 等人 ${ }^{[45]}$ 的观点. 类似灵井遗址这样的多个动 物属种、多个动物个体以及大量伴生石制品同时出现 的考古类遗址未必就有“居址”或“居住面”方面的性 质与功能. 从灵井遗址的实际情况分析, 相信这一动 物群实际上还是代表了一种狩猎-屠宰行为的集合, 而与“居址”或“生活面”等概念相去甚远.

至于遗址中相对较多的石制品, 则可以从民族 学的材料中得到一些启发. O'Connell 等人 ${ }^{[34]}$ 曾经描 述了现代 Hadza 狩猎采集群体在执行潜伏式狩猎任 务时往往会在猎物最容易出现的位置附近搭建或者 寻找一个藏身之所, 然后他们就可以在等待猎物出 现的过程中进行其他活动，比如制作、修理工具等; 类似这样的活动无疑将会增加遗址中石制品的数量. 此外, 在一定的生态和地理条件下, 当狩猎者可以预 期未来不远的狩猎及肢解猎物活动时 (比如在类似灵 井遗址这样的湖滨、水畔环境), 他们可能会将部分 制作石制品的原料或者是已经完成的石制品“存储” 在附近，从而也使此类遗址一定程度上具备了“存储 仓库”的功能, 因此也将不可避免地增加遗址中石制 品的数量. 事实上, 在目前已获广泛认可的“狩猎-屠 宰遗址”中，一般都有数千甚至上万件石制品的出
现 $^{[45,46]}$, 这与 $\mathrm{Issac}^{[5]}$ 经典模型中的遗址特点已经相 去甚远. 综上所述, 我们相信, 以功能与性质而言, 灵井遗址无疑还应属于“B 类遗址”, 只是其考古内涵 更为丰富一些而已.

相对于旧石器时代考古记录中更为常见的“居 址”而言, 狩猎-屠宰遗址内的古人类活动性质相对单 一, 但却是我们了解和阐释古人类生存手段、社会组 织乃至群体行为的重要补充和依托. 在灵井遗址这 样的以古湖泊或古泉水为中心的古人类活动地点附 近，狩猎者熟悉并合理利用了遗址周边的地形地貌 条件, 并以群体合作等组织形式成功地将原始牛、普 通马等大型猎物驱赶至湖水的一定深度之内 ${ }^{[47,48]}$ 这一策略较为有效地降低了猎物的行动速度, 从而 保证了狩猎活动的顺利进行.

\section{5 结论}

灵井动物群中的动物属种分布及丰度、骨骼单元 分布、骨骼表面改造痕迹与死亡年龄模式等埋藏学证 据指示了古人类狩猎、肢解行为的存在, 同时也确立 了古人类在这一动物群聚集、改造(如肢解、剔肉等 人类生存活动)过程中的主导性地位. 基于这一动物 群的埋藏学特点及动物考古学证据, 并结合生态环 境等方面的线索, 将灵井许昌人遗址解释为旧石器 时代中期的狩猎-屠宰遗址, 这是迄今为止中国北方 旧石器时代中期首个经过系统埋藏学分析确认的此 类考古遗址. 灵井遗址中数量较多的人工石制品的 出现, 可能反映了这一时期古人类在生存策略和行 为活动方面的计划性和前瞻性(如对湖滨等临水环境 狩猎行动的预期以及石制品的存储行为等). 益的建议, 河南省文物考古研究所的孙新民所长至始至终关心并支持研究工作的顺利进行, 作者在此一并致谢.

\section{参考文献}

1 Lewin R, Foley R A. Principles of Human Evolution. 2nd ed. Oxford: Blackwell Publishing, 2004

2 Bunn H T, Kroll E M. Systematic butchery by Plio-Pleistocene hominids at Olduvai Gorge, Tanzania. Curr Anthropol, 1986, 27: 431-452

3 Binford L R. Nunamiut Ethnoarchaeology. New York: Academic Press, 1978

4 Bartram L E. Perspectives on skeletal part profiles and utility curves from eastern Kalahari ethnoarchaeology. In: Hudson L, ed. From Bones to Behavior: Ethnoarchaeological and Experimental Contributions to the Interpretation of Faunal Remains. Illinois: Center for Archaeological Investigations, 1993. 115-137

5 Isaac G L, Crader D C. To what extent were early hominids carnivorous? An archaeological perspective. In: Hardinger R S O, Teleki G, eds. Omnivorous Primates. New York: Columbia University Press, 1981. 37-103 
6 Lyman R L. Vertebrate Taphonomy. Cambridge: Cambridge University Press, 1994

7 Gifford D P. Taphonomy and paleoecology: A critical review of archaeology's sister disciplines. In: Schiffer M B, ed. Advances in Archaeological Method and Theory, Vol.4. New York and London: Academic Press, 1981. 365-438

8 Domínguez-Rodrigo M, Barba R, Egeland C P. Deconstructing Olduvai: A Taphonomic Study of the Bed I Sites. New York: Springer, 2007

9 Domínguez-Rodrigo M, Pickering T R, Semaw S, et al. Cutmarked bones from Pliocene archaeological sites at. Gona, Afar, Ethiopia: Implications for the function of the world's oldest stone tools. J Hum Evol, 2005, 48: 109-121

10 李占扬. 许昌灵井遗址 2005 年出土石制品初步研究. 人类学学报, 2007, 26: 138-153

11 李占扬, 董为. 河南许昌灵井旧石器遗址哺乳动物群的性质及时代探讨. 人类学学报, 2007, 26: 345-360

12 Gao X, Norton C J. A critique of the Chinese "Middle Palaeolithic". Antiquity, 2002, 76: 397-412

13 Norton C J, Gao X, Feng X W. The East Asian middle paleolithic reexamined. In: Camps M, Chauhan P R, eds. Sourcebook of Paleolithic Transitions: Methods, Theories, and Interpretations. New York: Springer, 2010. 245-254

14 张双权. 河南许昌灵井动物群的埋藏学研究. 博士学位论文. 北京: 中国科学院研究生院, 2009

15 Brain C K. The Hunters or the Hunted? An Introduction to African Cave Taphonomy. Chicago: University of Chicago Press, 1981

16 Isaac G L. The archaeology of human origins. Adv World Archaeol, 1984, 3: 1-87

17 Norton C J, 张双权, 张乐, 等. 上/更新世动物群中人类与食肉动物“印记”的辨识. 人类学学报, 2007, 26: 183-192

18 Andrews P. Owls, Caves, and Fossils: Predation, Preservation, and Accumulation of Small Mammal Bones in Caves, with An Analysis of the Pleistocene Cave faunas from Westbury-sub-Mendip, Somerset, UK. Chicago: University of Chicago Press, 1990

19 Sanders W J, Trapani J, Mitani J C. Taphonomic aspects of crowned hawk-eagle predation on monkeys. J Hum Evol, 2003, 44: 87-105

20 Behrensmeyer A K. Taphonomy and hunting. In: Nitecki M H, Nitecki D V, eds. The Evolution of Human Hunting. New York: Plenum Press, 1987. 423-450

21 Behrensmeyer A K. Bones through time: The importance of biotic versus abiotic taphonomic processes in the vertebrate fossil record. In: Renzi D, Alonso M, Belinchon M, et al, eds. Current Topics on Taphonomy and Fossilization. Valencia: Proceedings of the International Conference Taphos, 2002. 297-304

22 Potts R. Early Hominid Activities at Olduvai Gorge. New York: Adline de Gruyter, 1988

23 Hanks J. The Struggle for Survival. New York: Mayflower Books, 1979

24 Haynes G. Longitudinal studies of african elephant death and bone deposits. J Archaeol Sci, 1988, 15: 131-157

25 Berger J. Ecology and catastrophic mortality in wild horses: Implications for interpreting fossil assemblages. Science, 1983, 220: $1403-1404$

26 Sinclair A R E. The African Buffalo. Chicago: University of Chicago Press, 1977

27 Cruz-Uribe K. Distinguishing hyena from hominid bone accumulations. J Field Archaeol, 1991, 18: 467-486

28 Lacruz R, Maude G. Bone accumulations at brown hyena (Parahyaena brunnea) den sites in the Makgadikgadi Pans, Northern Botswana: Taphonomic, behavioral and palaeoecological implications. J Taphonomy, 2005, 3: 43-54

29 Selvaggio M M. The archaeological implications of water-cached hyena kills. Curr Anthropol, 1998, 39: 380-383

30 张双权, 李占扬, 张乐, 等. 河南灵井许昌人遗址大型食草类动物死亡年龄分析及东亚现代人类行为的早期出现. 科学通报, 2009 , 54: $2857-2863$

31 Steele T E. Red deer: Their ecology and how they were hunted by Late Pleistocene hominids in Western Europe. Doctor Dissertation. Stanford: Stanford University, 2002

32 Klein R G. The Human Career. 3rd ed. Chicago: University of Chicago Press, 2009

33 Speth J D. Hunting pressure, subsistence intensification, and demographic change in the levantine Late Middle Paleolithic. In: Goren-Inbar N, Speth J D, eds. Human Paleoecology in the Levantine Corridor. Oxford: Oxbow Press, 2004. 149-166

34 O'Connell J F, Hawkes K, Jones B. Patterns in the distribution, site structure, and assemblage composition of Hadza kill-butchering sites. J Archaeol Sci, 1992, 19: 319-345

35 张乐, Norton C J, 张双权, 等. 量化单元在马鞍山遗址动物骨骼单元研究中的运用. 人类学学报, 2008, 27: 79-90

36 Domínguez-Rodrigo M. Meat-eating by early hominids at the FLK 22 Zinjanthropus site, Olduvai Gorge, Tanzania: An experimental approach using cut mark data. J Hum Evol, 1997, 33: 669-690

37 Lupo K D, O'Connell J F. Cut and tooth mark distributions on large animal bones: Ethnoarchaeological data from the Hadza and their implications for current ideas about early human carnivory. J Archaeol Sci, 2002, 29: 85-109

38 李占扬, 沈辰. 微痕观察初步确认灵井许昌人遗址旧石器时代骨制工具. 科学通报, 2010, 55: 895-903

39 Binford L R. In pursuit of the past: Decoding the archaeological record. California: University of California Press, 1983

40 Andresen J M, Byrd B F, Elson M D, et al. The Deer Hunters: Star Carr Reconsidered. World Archaeol, 1981, 13: 31-46 
41 Pitts M. Hides and antlers: A new look at the gatherer-hunter site at Star Carr, North Yorkshire. World Archaeol, 1979, 11: 32-42

42 Bunn H T. Early Pleistocene hominid foraging strategies along the ancestral Omo River at Koobi Fora, Kenya. J Hum Evol, 1994, 27: 247-266

43 Domínguez-Rodrigo M. Butchery and kill sites. In: Pearsall D M, ed. Encyclopedia of Archaeology. California: Academic Press, 2008. 948-953

44 Fiore L, Bondioli A, Coppa R, et al. Taphonomic analysis of the Late Early Pleistocene bone remains from Buia (Dandiero Basin, Danakil Depression, Eritrea): Evidence for large mammal and reptile butchering. In: Abbate E, Woldehaimanot Y, Libsekal Y, et al, eds. A Step Towards Human Origins: The BuiaHomo One-Million-Years Ago in the Eritrean Danakil Depression (East Africa). Milano: Dipartimento di Science della Terra, 2004. 89-97

45 Chazan M, Horwitz L K. Finding the message in intricacy: The association of lithics and fauna on lower paleolithic multiple carcass sites. J Anthropol Archaeol, 2006, 25: 436-447

46 Delagnes A, Lenoble A, Harmand S, et al. Interpreting pachyderm single carcass sites in the African Lower and Early Middle Pleistocene record: A multidisciplinary approach to the site of Nadung'a 4 (Kenya). J Anthropol Archaeol, 2006, 25: 448-465

47 Blehr O. Communal hunting as a prerequisite for caribou (wild reindeer) as a human resource. In: Leslie B, Davis L B, Reeves B, eds. Hunters of the Recent Past. London: Unwin Hyman, 1990. 304-326

48 Voormolen B. Ancient hunters, modern butchers: Schöningen 13II-4, a kill-butchery site dating from the northwest European Lower Palaeolithic. Dissertation for the Doctoral Degree. Leiden: Leiden University, 2008 